\title{
ON THE EXISTENCE OF ADDITIONAL ZERO TEMPERATURE PHASE TRANSITIONS IN SU(2) LATTICE GAUGE THEORY
}

\author{
J. ENGELS and J. SEIXAS ${ }^{1}$ \\ Fakultät für Physik, Universität Bielefeld, D-4800 Bielefeld, Fed. Rep. Germany
}

Received 19 February 1988

\begin{abstract}
We show that the recently observed third order zero temperature deconfining transition at $4 / g^{2} \approx 2.475$ (and maybe at $4 / g^{2} \approx 2.56$ ) is non-existing; we argue that its discovery is based only on insufficient statistics.
\end{abstract}

The phase structure of $\mathrm{SU}(2)$ pure gauge theory is certainly one of the best studied subjects in the realm of lattice gauge theory. This is related to the fact that this simple model presents many characteristics required by QCD, with the major advantage of allowing for good statistical results within a reasonable amount of computer time.

The common lore about the phase structure of SU (2) pure gauge theory $[1,2]$ is that it exhibits a relatively rapid crossover from a region well described by a weak coupling expansion to a region well characterized by a strong coupling expansion. A nonzero string tension is detectable all along this crossover region which, together with the observation of an exponential dependence of the inverse coupling constant in the weak coupling region [1] enforced the belief that confinement was a basic feature of this theory.

However, recently some doubts have been shed over this picture. First, Gribov [3] presented an argument according to which the renormalized coupling could tend to a finite value with increasing distance, that is, that there could be an infrared fixed point of the renormalization group $\beta$ function for a finite value of the coupling. This seems to imply the existence of an additional critical point. Secondly,

\footnotetext{
Work supported by the Deutsche Forschungsgemeinschaft, under research grants En 164/2 and Pe 340/1-2.

1 On leave of absence from CFMC/INIC, P-1699 Lisbon, Portugal.
}

Grady [4] has suggested, by studying numerically the (pseudo) specific heat

$C=-\beta^{2}(\partial / \partial \beta) P$,

$P=1-\frac{1}{2}\left\langle\operatorname{Tr} U_{\mathrm{p}}\right\rangle$,

where $\beta=4 / g^{2}$ and $P$ is the plaquette average or the internal energy, that there should be a third order zero temperature phase transition at $\beta \approx 2.475$. This author also suggested that the existence of this phase transition might be an explanation for the apparent lack of asymptotic scaling in the crossover region [5]. Finally, the possibility of additional critical points has also been more considered by other authors [6].

It is important to emphasize that if the string tension (or - what amounts to the same - the correlation length ) is the only scale of the pure gauge theory, then it must vanish at any critical point of the theory. The existence of a phase transition at some finite value of the coupling implies then that the theory does not confine for all values of the coupling constant i.e., this transition would be a deconfining one. It clearly is of the utmost importance to decide on the existence or non-existence of such a phase transition. The purpose of this letter is to show that there is no indication whatsoever for such a phase transition in the region of couplings proposed in ref. [4].

To compute plaquette averages on $12^{4}$ and $16^{4}$ lattices we have used a vectorized full group heatbath program, which took 5.6 $\mu$ s per link on a CYBER 205. For the thermalization we discarded the first 1000 
sweeps after an ordered start at each $4 / g^{2}$ value. Our results are presented in table 1 . We found that if the number of sweeps is about 2000 on a $12^{4}$ lattice the error in the plaquette value is of the order 0.0003 , i.e. not even the fourth digit of $P$ is known. If one intends to calculate the specific heat from $P$ by building differences in $P$ and dividing by the corresponding $\Delta \beta$, then of course a much higher accuracy is needed. Therefore, we have performed 35000-75000 (at $\beta=2.475$ and 2.55 - the "critical" points -95000 ) iterations on the $12^{4}$ and $15000-30000$ iterations on the $16^{4}$ lattice. That reduces the errors on the average plaquette values to $(4-8) \times 10^{-6}$. There is still a finite size difference between the $12^{4}$ and $16^{4}$ lattice results, the main part of which we can take away by subtracting the known $\mathrm{O}\left(g^{4}\right)$ weak coupling expansions [7] on the two lattices from $P$

$P_{\mathrm{r}}=P-P_{\mathrm{wc}}$,

where

$P_{\mathrm{wc}}^{(12)}=0.749964 / \beta+0.1513479 / \beta^{2}$,

Table 1

Table for plaquette values. $N$ represents the number of measurements.

\begin{tabular}{lllll}
\hline & $4 / g^{2}$ & $P$ & $\begin{array}{l}\Delta P \\
\left(\times 10^{5}\right)\end{array}$ & \\
\hline $12^{4}$ & & & & \\
& 2.4500 & 0.3583943 & 0.64 & 48300 \\
& 2.4625 & 0.3556966 & 0.75 & 34000 \\
& 2.4750 & 0.3530633 & 0.44 & 94000 \\
& 2.4875 & 0.3505094 & 0.72 & 34000 \\
2.5000 & 0.3480089 & 0.57 & 54000 \\
2.5250 & 0.3431429 & 0.48 & 74000 \\
2.5500 & 0.3385481 & 0.41 & 94000 \\
2.5750 & 0.3341388 & 0.45 & 78000 \\
2.6000 & 0.3299055 & 0.45 & 74000 \\
2.6250 & 0.3258098 & 0.44 & 74000 \\
2.6500 & 0.3218713 & 0.64 & 34000 \\
& 2.4000 & 0.3699898 & 0.70 & 14000 \\
2.4250 & 0.3640542 & 0.68 & 14000 \\
2.4500 & 0.3584122 & 0.67 & 14200 \\
2.4750 & 0.3530839 & 0.65 & 14000 \\
2.5000 & 0.3480464 & 0.46 & 26000 \\
2.5250 & 0.3432319 & 0.62 & 14000 \\
2.5500 & 0.3386428 & 0.42 & 29000 \\
2.5750 & 0.3342358 & 0.59 & 14000 \\
2.6000 & 0.3299673 & 0.40 & 29000 \\
2.6250 & 0.3258785 & 0.57 & 14000 \\
\hline
\end{tabular}

$P_{\mathrm{wc}}^{(16)}=0.749988 / \beta+0.1513711 / \beta^{2}$.

In fig. 1 we show these reduced plaquette values calculated from our data. The curves are smooth and show no structure which might indicate a transition. The specific heat

$C_{\mathrm{r}}=C-C_{\mathrm{wc}}$,

which was calculated from the $P_{\mathrm{r}}$-values by difference quotients is plotted in fig. 2 . To guide the eye we have connected the $16^{4}$ results by straight lines; the $12^{4}$ points are seen to agree very well with the $16^{4}$ results. Again no structure is seen. If we plot our $12^{4}$ averages from the first 10000-15000 iterations, we may also discover structures in $C_{\mathrm{r}}$, which however disappear with increasing statisics. For comparison we have included in fig. 2 the $C_{\mathrm{r}}$ values as obtained from the plaquette averages of Campostrini et al. [8] on a $16^{4}$ lattice, which were cited by Grady [4] in support of his transition hypothesis. Because of their low statistics ( 300 thermalization sweeps, 200 measurement sweeps) the data from ref. [8] show of course some noisy structure, which is however irrelevant.

To further support our findings we show in figs. 3 and 4 the the plaquette distributions at the "critical" points $4 / g^{2}=2.475$ (on the $12^{4}$ lattice) and

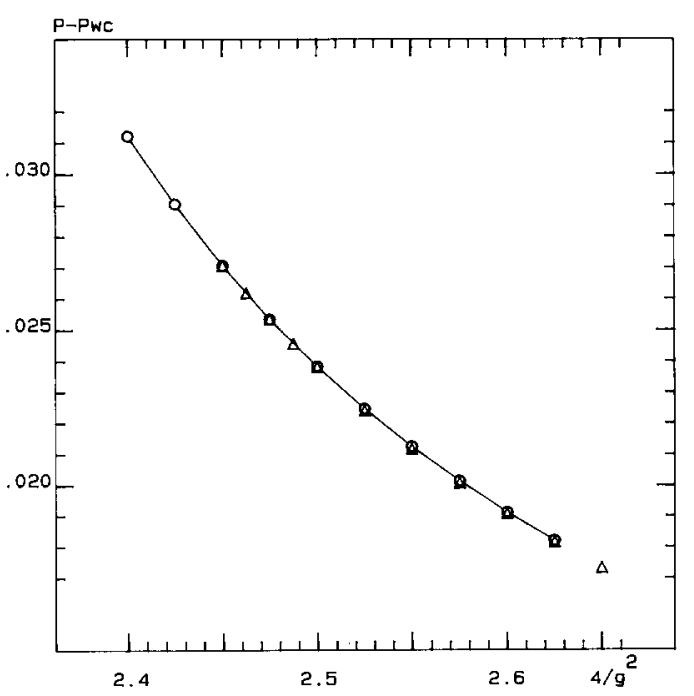

Fig. 1. Reduced plaquette values on $12^{4}(\Delta)$ and $16^{4}(O)$ lattice versus $4 / g^{2}$. The circles have been connected by straight lines to guide the eye. 


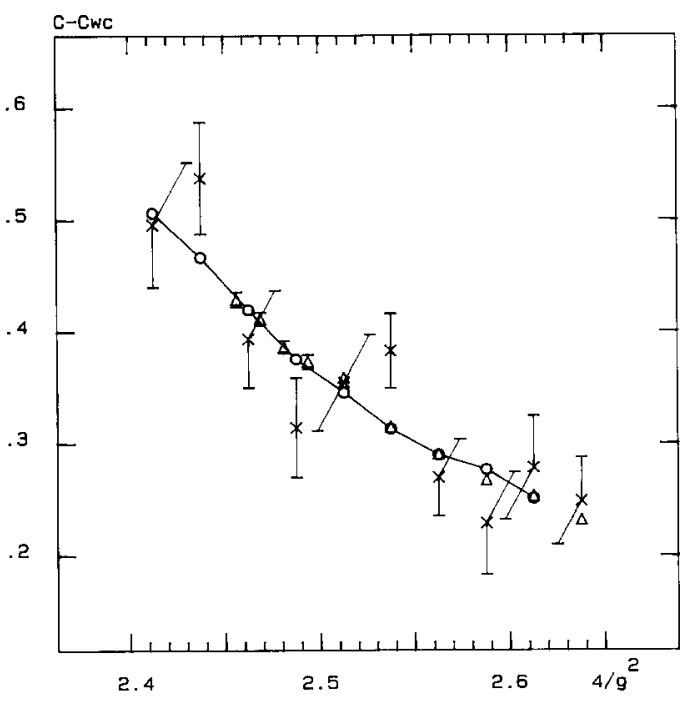

Fig. 2. Reduced specific heat versus $4 / g^{2}$; the notation is like in fig. 1 . The values of ref. [8] are denoted by crosses.

$4 / g^{2}=2.55$ (on the $16^{4}$ lattice) together with gaussian fits. Evidently, there is no indication to any transition since the distributions are well represented by gaussians.

Let us finally comment on the possibility that Wilson line measurements on asymmetric lattices [9]

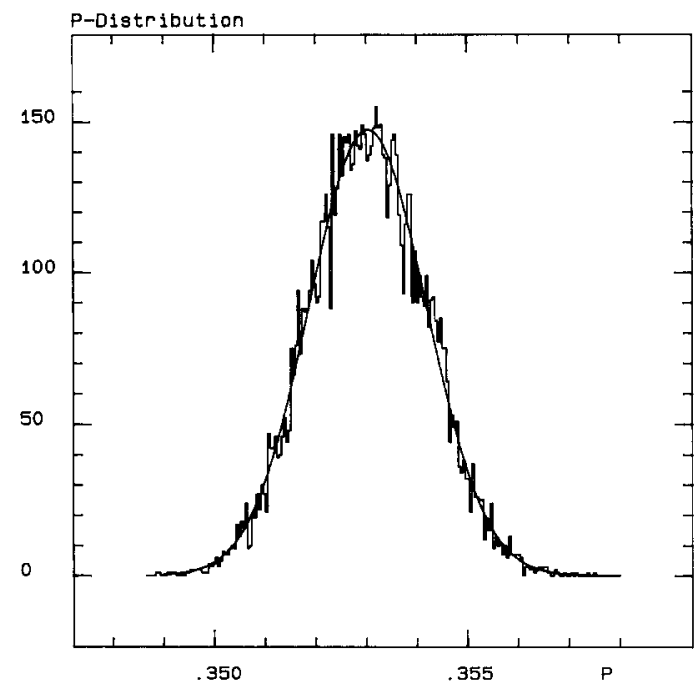

Fig. 3. The frequency distributions for the plaquette values at $4 / g^{2}=2.475$. The plot contains every tenth measurement of a 94000 iterations run on a $12^{4}$ lattice. The line is a gaussian fit to the histogram.

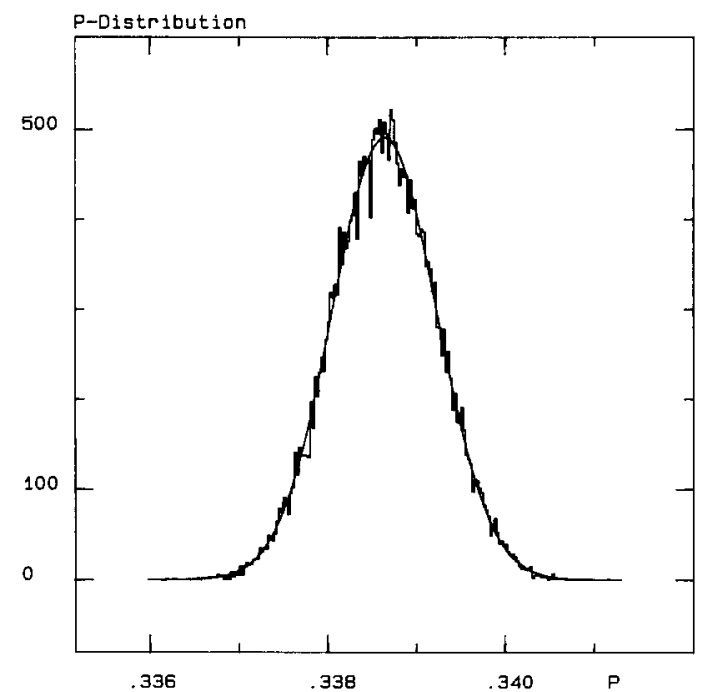

Fig. 4. The frequency distributions for the plaquette values at $4 / g^{2}=2.55$. The plot contains all measurements of a 29000 iterations run on a $16^{4}$ lattice. The line is a gaussian fit to the histogram.

support the existence of the "critical" point at $\beta=2.475$ and do not show the usual finite temperature phase transition. It was argued in ref. [4] that on a rather large spatial volume lattice (here $18^{3}$ ) the Wilson line average below the transition point should be essentially zero. As is well known [10], on a finite lattice there is no true order parameter. It is usually approximated by taking the average of the modulus of the lattice average of the Wilson line, which of course never becomes zero on a finite lattice, even below the transition. The Wilson line average itself would become zero everywhere, also above the transition, if enough iterations would be performed. Apart from that, the ratio of the number of points in space to the one in time direction determines whether a volume is relatively large or not and whether the deconfinement transition point can be easily identified.

We have shown that there is no evidence for a third order zero temperature phase transition for SU(2) lattice gauge theory in the range $2.45<\beta<2.60$. To prove the existence of such a transition is always very difficult. Data with low statistics will, of course, always exhibit some structure. If one does not check then that this structure persists or even becomes stronger with much higher statistics, any conclusion on these data remains premature. 


\section{References}

[1] M. Creutz, Phys. Rev. D 21 (1980) 2308

[2] M. Creutz, Phys. Rev. Lett. 43 (1979) 553; 45 (1980) 513.

[3] V.N. Cribov, preprint KFKI-1986-29-A (June 1986); Phys. Lett. B 194 (1987) 119.

[4] M. Grady, preprint ANL-HEP-PR-87-12, Z. Phys. C, to be published.

[5] F. Karsch and C.B. Lang, Phys. Lett. B 138 (1984) 176;

F. Gutbrod and I. Montvay, Phys. Lett. B 136 (1984) 411;

U. Heller and F. Karsch, Phys. Rev. Lett. 54 (1985) 1765;

A. Patel and R. Gupta, Nucl. Phys. B 251 [FS13] (1985) 789 ;
F. Gutbrod, Z. Phys. C 30 (1986) 585; Phys. Lett. B 186 (1987) 389

[6] A. Patrascioiu, E. Seiler and I.O. Stamatescu, preprint MPIPAE/PTh 75/87 (September 1987).

[7] F. Karsch and U. Heller, Nucl. Phys. B 251 [FS13] (1985) 254.

[8] M. Campostrini, G. Gurci, A. DiGiacomo and G. Paffuti, Z. Phys. C 32 (1986) 377.

[9] J. Engels, J. Jersák, K. Kanaya, E. Laermann, C.B. Lang, T. Neuhaus and H. Satz, Nucl. Phys. B 280 [FS18] (1987) 577.

[ 10] See e.g. J. Engels, Proc. 1987 Seillac Conf. on Lattice gauge theories. 